\title{
The Role of Probiotic Supplementation on Insulin Resistance in Obesity- Associated Diabetes: A Mini-Review
}

\author{
Seeme Saha ${ }^{1}$ Nazmun Nahar Alam ${ }^{2}$ S M Niazur Rahman ${ }^{3 *}$ \\ ${ }^{1}$ Research Assistant, Center for Biomedical Research and Training (CBRT), Dhaka, \\ Bangladesh \\ Email: seeme@cbrtrain.org \\ ${ }^{2}$ Lecturer, Faculty of Medicine, AIMST University, Kedah, Malaysia \\ Email: nazmun1749@gmail.com, ORCID id: 0000-0003-2408-7190 \\ ${ }^{3}$ Executive Director, Center for Biomedical Research and Training (CBRT), Dhaka, \\ Bangladesh \\ Email: niazur@cbrtrain.org, ORCID id: 0000-0002-0584-0792 \\ * Corresponding Author:

\section{Dr. S M Niazur Rahman} \\ Executive Director, Center for Biomedical Research and Training (CBRT) \\ House-113, Road-04, Block-F, Journalist R/A, Mirpur, Dhaka, Bangladesh. \\ Email: niazur@cbrtrain.org, niazur03@gmail.com
}

\begin{abstract}
Background: Obesity and diabetes are two metabolic disorders linked by an inflammatory process named insulin resistance (IR). Various research on the role of gut microbiota in developing obesity and its associated disorders has led to the growing interest in probiotic supplementation. Considering the life-threatening complications of diabesity this mini review explored the effects of probiotic supplementation on IR in obesity associated diabetes. Method: This review is based on recent articles from 2005-2020, studying the role of probiotic supplementation on glucose and insulin parameters in healthy and diabetic mouse model. Result: Probiotic supplementation altered the gut microbiota composition, increased short chain fatty acid production, and decreased pro inflammatory cytokines. Additionally, they decreased intestinal permeability, circulating lipopolysaccharides and metabolic endotoxemia, hence improved insulin sensitivity and reduced obesity. Although multi-strain probiotic supplementation showed greater benefits than single strain interventions, variations in the concentration of probiotics used and the duration of treatment also influenced the results. Conclusion: Probiotic supplementation could manipulate the gut microbiota by reducing intestinal permeability, inflammation and ameliorate IR and obesity associated diabetes in animal models which requires further long-term clinical studies in humans.
\end{abstract}

Key Words: Probiotics, Gut microbiota, Obesity, Insulin resistance, Type 2 Diabetes. 


\section{Introduction}

Diabesity refers to the coexistence of two metabolic disorders; type 2 diabetes (T2D) and obesity, the present epidemic requires immediate global attention ${ }^{1}$. Contemporary lifestyle involving declined physical activity and increased calorie intake results in this chronic disorder. ${ }^{2}$ The worldwide dual epidemic of obesity and T2D is increasing at an alarming rate. At present 463 million adults worldwide are diabetic, and the number is expected to rise to 700 million by $2045 .^{5}$ Adding to the risk of developing diabesity, WHO stated that the number of obese people worldwide has tripled since 1975 with a total being 650 million in $2016 .{ }^{6}$ T2D, a multifactorial disease results hyperglycaemia due to an absolute or relative lack of insulin or cellular resistance to insulin, the predominant factors contributes its development are genetic mutations and environment. ${ }^{3}$ Moreover, inability to produce insulin from pancreatic beta cells and peripheral IR, results in perturbation of glucose homeostasis leading to development of obesity and its associated disorders. IR gets accelerated by obesity when accompanied by pancreatic $\beta$-cells dysfunction results in the development of T2D. ${ }^{3}$ Hence, obesity can be reported as a major risk factor for the development of IR and T2D. ${ }^{4}$

Modification of the gut microbiota has been studied to play a vital role in ameliorating obesity and its associated disorders. Gut microbiota is a complex community of microorganisms, the major phylae being inhabit in our gastrointestinal tract are Firmicutes, Bacteroidetes, Actinobacteria, Proteobacteria and Verrucomicrobia. ${ }^{7}$ Their main functions are to extract energy from indigestible food, synthesize essential vitamins and modulate immune system ${ }^{8}$ Dysbiosis of these gut microbes can dysregulate energy metabolism, gut permeability, inflammation and ultimately lead to conditions like IR, obesity and T2D. ${ }^{3}$ Bagarolli et al. found that probiotic-treated mice receiving a high fat diet (HFD) gained significantly less weight and had reduced food intake compared with animals that did not receive probiotics. The metagenomic sequencing of feces of experimental mice shows that supplementing Bifidobacterium as probiotic has increased the gut concentration of Bifidobacterium and decreased the concentration of Firmicutes which is associated with the development of T2D. ${ }^{8}$

IR is a pathological condition of declined cellular response to insulin causing hyperglycaemia, despite of $\beta$-cells of the pancreas are being able to produce enough insulin. This state of cellular non-responsiveness to insulin characterizes the transition from IR to T2D. ${ }^{9}$ In obese individuals the adiposity of body increases and so does the free fatty acid. Free fatty acid blunts the $\mathrm{PI} 3 \mathrm{~K} / \mathrm{AKT} / \mathrm{mTOR}$ pathway leading to decreased insulin sensitivity. ${ }^{8}$ Reactive oxygen species (ROS) produced from $\beta$-oxidation of saturated fat increases gut permeability causing leaky gut .$^{3}$ This leads to the activation of TLR signaling pathway and production of pro-inflammatory cytokines like TNF- $\alpha$ and IL-1 $\beta$, IL- 6 , causing development of IR. ${ }^{8}$ However, probiotics 
reverse the process by decreasing the activation of TLR signaling pathway and thus reducing production pro-inflammatory cytokines. ${ }^{8}$

Probiotics have been used as an interventional approach for decades. Their role in the modification of gut microbiota was described 500 years ago, but their clinical implementation is being studied for not more than 50 years. ${ }^{10}$ Probiotic supplementation has been studied by single and multiple strain administration, under various conditions to analyse their effect individually and in combination. Though studies show that multi-strain supplementation has more beneficial effects than single strain, ${ }^{11}$ many other factors like the concentration and duration of treatment also influences the result. The commonly used probiotics are Bifidobacterium, Lactic acid bacteria (LAB), dairy Propionibacterium, yeasts (Saccharomyces boulardii), Bacillus and E. coli. ${ }^{12}$ They are known to modulate the body's immune system through cellular components like polysaccharides, peptidoglycans, DNA etc. Probiotics increase satiety and glucose tolerance by decreasing leptin signalling and modulating AMPK pathways. ${ }^{13}$ They also restore the expression of tight junction proteins like Zolin-1 and Occludin to reduce intestinal permeability. ${ }^{8}$

Obesity and insulin resistance run parallelly, where one can be the cause of developing the other. Both being responsible for the development of T2D are strongly interconnected. However, probiotics add to this relationship by altering the gut microbiota and reducing obesity, IR and T2D. In this review we analyzed the recent studies to overview the effects of probiotic supplementation on IR in obesity associated T2D.

\section{Methods}

This review was performed based on original and review articles available on the electronic database of PubMed from the National Library of Medicine. The Medical Subject Headings (MeSH) used for the search were: 'probiotics', 'gut microbiota', 'obesity', 'Type 2 Diabetes' and 'insulin resistance' in various combinations. Thirteen different articles were initially found from the PubMed search. Three independent reviewers analyzed the search results by reading the titles, abstracts, and objectives. Disagreements were solved through meetings and discussions. The overall records identified by common acceptance were then narrowed down based on inclusion criteria. The articles selected were the ones with an objective of determining the effects of probiotic supplementation on IR in obesity-associated diabetes. The exclusion criteria were clinical trials, gestational diabetes, Type 1 diabetes, and lack of adequate information regarding the use of probiotics. Our review articles mostly included recent studies ranging from 2005-2020. Probiotics used in our review paper were various strains of Lactobacillus, Bifidobacterium and Akkermansia on both diabetic and healthy mice. They were 
subjected to high fat diet in a controlled environment with administration of single or multistrain probiotics.

\section{Results and Discussion}

Table 1.0 includes the effect of probiotic supplementation on glucose metabolism and IR. Results from seven different articles have been tabulated and interpreted. A. muciniphila reduced hepatic glycogen, inflammatory markers, improved mucosal layer thickness and thus reduced metabolic endotoxemia and IR. ${ }^{14,15}$ Supplementation of Lactobacillus casei increased insulin sensitivity and glucose tolerance by reducing TNF- $\alpha$ and other inflammatory markers. This reduced pancreatic cell degeneration and induced regeneration. Firmicutes-toBacteroidetes ratio, a suggested marker of metabolic disorders and T2D decreased. ${ }^{16}$ Bifidobacterium pseudocatenulatum showed an improvement in the glucose profile and decreased serum leptin concentration. ${ }^{17}$ L. rhamnosus used in combination with other strains and with Bifidobacterium promoted the release of glucagon-like protein 1(GLP-1), resulting in reduced food intake and improved glucose tolerance. ${ }^{8,18}$ It has also elevated the expression tight junction proteins and reduced LPS translocation and macrophage infiltration contributing to reduced endotoxemia and improved insulin sensitivity.

\section{Probiotics and their potential mechanism of action}

With the evolution of research, probiotics now contain microorganisms ranging from $10^{\wedge} 8$ $10^{\wedge} 10 .{ }^{10}$ Commonly used probiotics include large variety of Lactobacillus and Bifidobacterium species. ${ }^{19}$ Lactic acid bacteria (LAB) broadly used as probiotic represents a group of microorganisms found in fermented milk products and in the gastrointestinal and urinary tracts in animals. ${ }^{20}$ LAB produce bacteriocins that can directly modulate the gut microbiota. ${ }^{13}$ Propioni- bacterium, a non-LAB is known to produce metabolites such as short chain fatty acids (SCFA), vitamins B8, B9, B12 and bifidogenic anti-inflammatory products (DHNA). ${ }^{19}$ While the SCFAs help in maintaining the gut barrier integrity, ${ }^{21}$ anti-inflammatory products reduce IR. SCFA also stimulate the L-cells to produce glucagon-like-protien-1 (GLP-1), which improves glucose tolerance and reduce food intake. ${ }^{22}$ Butyrate maintains the intestinal barrier by acting as a nutrient for the gut colonocytes. ${ }^{23}$ The restored gut barrier prevents translocation of LPS, reducing metabolic endotoxemia and IR. ${ }^{8}$

\section{Probiotics, modulation of gut microbiota and energy metabolism}

Along with healthy eating and physical exercise, cultivating a diverse gut microbiome is important for controlling obesity and T2D. Recent studies have shown that the gut microbiome is more diverse in healthy individuals than in ones with T2D. ${ }^{24}$ This diversity can be brought about by probiotics supplementation, either through fermented food and beverages like yogurt, cheese ${ }^{25}$ or through dietary supplements in the form of tablets and capsules ${ }^{26,27}$. Yogurt, a 
fermented milk product containing probiotics when consumed was seen to reduce weight, inflammatory markers, and the risk of developing T2D. ${ }^{28}$ Microorganisms such as Bacteroidetes and Actinobacteria which have been studied to be negatively associated with T2D was increased and the prevalence of Firmicutes which is positively associated with T2D decreased with probiotic supplementation. ${ }^{22,29}$ Observations also suggest that differences in gut microbiota composition varied according to the body's adiposity. In a study, prevalence of Firmicutes was found to be higher in obese mice than in lean mice. ${ }^{30}$ Modulation of the gut microbiota causes alterations of different genes and pathways to regulate energy metabolism. Probiotics have been studied to suppress the expression of AMP-activated protein kinase (AMPK), which decreased oxidation of fatty acid in muscles leading to increased insulin sensitivity. ${ }^{31}$

\section{Probiotics: dose, duration, single and multi-strain intervention}

In our review we explored the effects of probiotic supplementation along with the effective doses and duration for their optimum functioning. Wang et al. ${ }^{16}$ studied that administration of Lactobacillus case $i$ in a concentration of $10^{\wedge} 8-10^{\wedge} 9$ CFU showed better improvement in IR when compared with the concentration of $10^{\wedge} 10 \mathrm{CFU}$. This shows that administration of an appropriate dose of probiotic is essential for its maximum efficacy. Duration of probiotic supplementation also plays an important role in attenuating obesity associated disorders. Probiotic supplementation did not have any changes in the insulin and glucose profiles at 3rd and 4 th week ${ }^{32,15,18}$ until the $16^{\text {th }}$ week in an experiment carried out for 24 weeks on HFD induced mice using different strains of Lactobacillus. Just like dose and duration, single and multi-strain probiotic administration can vary the results. Multi-strain probiotic shows greater benefits on metabolic health than single strain probiotic supplementation. For example, single strain of Bifidobacterium used in a study ${ }^{17}$ showed much less benefits in improving metabolic health compared to the study performed by Bagarolli et al. who used two different strains of Lactobacillus in combination with Bifidobacterium. The multi-strain supplementation showed a marked reduction in inflammatory cytokine production and macrophage infiltration ${ }^{8}$ in contrast to the single strain supplementation. ${ }^{17}$

\section{Probiotics supplementation improves insulin resistance}

Obesity causes low grade systemic inflammation by visceral adipocytes releasing cytokines like leptin, adiponectin, and inflammatory cytokines like tumor necrotic factor- $\alpha$ (TNF- $\alpha$ ), interleukin-6 (IL-6). This elevated inflammation and subsequent cytokine release alter insulin signaling and contribute to IR. ${ }^{9}$ Furthermore, saturated fatty acids (FAs) and alterations in gut microbiota triggers metabolic inflammation by stimulating pattern-recognition receptors (PRRs) like toll-like receptors (TLR), nucleotide oligomerization domain (NOD), and inflammasome. ${ }^{33,34}$ These eventually increase pro-inflammatory cytokines, T lymphocytes and macrophages that activate numerous kinases which interfere with insulin signaling and insulin 
action. ${ }^{9}$ Probiotic supplementation has been studied to reduce the expression of NOD1 and PRR genes in obese mice causing reduction in weight gain and IR. ${ }^{8}$ The protein kinases JNK and IKK responsible for inflammation and IR are also downregulated by probiotic supplementation .${ }^{8}$ Obesity has been studied to increase the expression of TLR4 in mice which negatively affects insulin sensitivity and cascade T2D. ${ }^{35}$ Probiotic supplementations reduce TLR signaling pathway, NFKB translocation into the nucleus and thus reduce inflammation and IR. ${ }^{8}$

\section{Probiotics alleviate diabesity}

Probiotics can alter the secretion of substances like leptin and adiponectin responsible for hypothalamic control of diet and glucose metabolism. ${ }^{8,22}$ Leptin, a hormone released from the adipose tissue causes hyperphagia. Probiotic supplementation in obese mice has been studied to decrease adipose tissue resulting in decreased leptin release, increasing STAT3 phosphorylation, POMC and thus reducing appetite.$^{8}$ A reduction in appetite causes decreased weight gain, fat mass accumulation, and hence reduces obesity. ${ }^{22}$ Obesity decreases the expression of GLUT-4 mRNA responsible for insulin sensitivity and glucose uptake. ${ }^{36}$ This is reversed by probiotic supplementation which increases serum GLP-1 levels and help regulate blood sugar and weight gain by acting on multiple receptors in the hypothalamus. ${ }^{8}$ Insulininduced $\mathrm{Akt}^{\wedge \mathrm{ser}} 473$ phosphorylation levels responsible for glucose metabolism, decreased with obesity is also recovered by probiotic supplementation. ${ }^{8}$ Thus, obesity induced IR and glucose intolerance which causes T2D is alleviated by probiotic supplementation.

\section{Conclusion}

Our data summarized that probiotic supplementation could manipulate the gut microbiota, reduce intestinal permeability and inflammation, leads to ameliorate IR and obesity associated diabetes in animal models. These findings warrant long term randomized clinical trials in human subjects. Despite the amplitude of this review, there were some limitations like the heterogeneity of the study samples, variation of concentration, dose and duration of probiotics used. However, probiotic supplementation in combination with lifestyle modification tends to treat and prevent metabolic disorders, which requires further investigation.

\section{Reference}

1. Pappachan JM, Fernandez CJ, Chacko EC. Diabesity and antidiabetic drugs. Molecular aspects of medicine. 2019 Apr 1;66:3-12.

2. Kumar N, Puri N, Marotta F, Dhewa T, Calabrò S, Puniya M, Carter J. Diabesity: an epidemic with its causes, prevention and control with special focus on dietary regime. Functional Foods in Health and Disease. 2017 Jan 31;7(1):1-6.

3. Wen L, Duffy A. Factors influencing the gut microbiota, inflammation, and type 2 diabetes. The Journal of nutrition. 2017 Jul 1;147(7):1468S-75S.

4. Eckel RH, Kahn SE, Ferrannini E, Goldfine AB, Nathan DM, Schwartz MW, Smith RJ, Smith SR. Obesity and type 2 diabetes: what can be unified and what needs to be 
individualized?. The Journal of Clinical Endocrinology \& Metabolism. 2011 Jun 1;96(6):1654-63.

5. Borgnakke WS. IDF Diabetes Atlas: Diabetes and oral health-A two-way relationship of clinical importance. Diabetes research and clinical practice. 2019 Nov 1;157:107839.

6. Obesity and Overweight, World Health Organization. Available at https://www.who.int/news-room/fact-sheets/detail/obesity-and-overweight accessed at 2021Jun 9.

7. Eckburg PB. Bik EM, Bernstein CN, Purdom E, Dethlefsen L, Sargent M, Gill SR, Nelson KE, Relman DA. Diversity of the human intestinal microbial flora [see comment]. Science. 2005;308:1635-8.

8. Bagarolli RA, Tobar N, Oliveira AG, Araújo TG, Carvalho BM, Rocha GZ, Vecina JF, Calisto K, Guadagnini D, Prada PO, Santos A. Probiotics modulate gut microbiota and improve insulin sensitivity in DIO mice. The Journal of nutritional biochemistry. 2017 Dec 1;50:16-25.

9. Wondmkun YT. Obesity, insulin resistance, and type 2 diabetes: associations and therapeutic implications. Diabetes, metabolic syndrome and obesity: targets and therapy. 2020;13:3611.

10. Wieërs G, Belkhir L, Enaud R, Leclercq S, Philippart de Foy JM, Dequenne I, de Timary P, Cani PD. How probiotics affect the microbiota. Frontiers in cellular and infection microbiology. 2020 Jan 15;9:454.

11. Salles BI, Cioffi D, Ferreira SR. Probiotics supplementation and insulin resistance: a systematic review. Diabetology \& metabolic syndrome. 2020 Dec;12(1):1-24.

12. Gareau MG, Sherman PM, Walker WA. Probiotics and the gut microbiota in intestinal health and disease. Nature reviews Gastroenterology \& hepatology. 2010 Sep;7(9):50314.

13. Anhê FF, Marette A, Le Barz M, Varin TV, Desjardins Y, Roy D, Levy E, Urdaci MC. Probiotics as Complementary Treatment for Metabolic Disorders. Diabetes \& Metabolism Journal. 2015 Aug 1;39(4).

14. Everard A, Belzer C, Geurts L, Ouwerkerk JP, Druart C, Bindels LB, Guiot Y, Derrien M, Muccioli GG, Delzenne NM, De Vos WM. Cross-talk between Akkermansia muciniphila and intestinal epithelium controls diet-induced obesity. Proceedings of the national academy of sciences. 2013 May 28;110(22):9066-71.

15. Wang J, Tang H, Zhang C, Zhao Y, Derrien M, Rocher E, Vlieg JE, Strissel K, Zhao L, Obin M, Shen J. Modulation of gut microbiota during probiotic-mediated attenuation of metabolic syndrome in high fat diet-fed mice. The ISME journal. 2015 Jan;9(1):1-5.

16. Wang G, Li X, Zhao J, Zhang H, Chen W. Correction: Lactobacillus casei CCFM419 attenuates type 2 diabetes via a gut microbiota dependent mechanism. Food \& function. 2017;8(10):3814-.

17. Moya-Perez A, Romo-Vaquero M, Tomas-Barberan F, Sanz Y, García-Conesa MT. Hepatic molecular responses to Bifidobacterium pseudocatenulatum CECT 7765 in a mouse model of diet-induced obesity. Nutrition, Metabolism and Cardiovascular Diseases. 2014 Jan 1;24(1):57-64.

18. Balakumar M, Prabhu D, Sathishkumar C, Prabu P, Rokana N, Kumar R, Raghavan S, Soundarajan A, Grover S, Batish VK, Mohan V. Improvement in glucose tolerance and insulin sensitivity by probiotic strains of Indian gut origin in high-fat diet-fed C57BL/6J mice. European journal of nutrition. 2018 Feb;57(1):279-95.

19. Rowland IR, Capurso L, Collins K, Cummings J, Delzenne N, Goulet O, Guarner F, Marteau P, Meier R. Current level of consensus on probiotic science-Report of an expert meeting-London, 23 November 2009: Report of an expert meeting-London, 23 November 2009. 
20. de LeBlanc AD, LeBlanc JG. Effect of probiotic administration on the intestinal microbiota, current knowledge and potential applications. World Journal of Gastroenterology: WJG. 2014 Nov 28;20(44):16518.

21. Brahe LK, Astrup A, Larsen LH. Can we prevent obesity-related metabolic diseases by dietary modulation of the gut microbiota?. Advances in nutrition. 2016 Jan;7(1):90101.

22. Yadav H, Lee JH, Lloyd J, Walter P, Rane SG. Beneficial metabolic effects of a probiotic via butyrate-induced GLP-1 hormone secretion. Journal of biological chemistry. 2013 Aug 30;288(35):25088-97.

23. Brahe LK, Astrup A, Larsen LH. Is butyrate the link between diet, intestinal microbiota and obesity-related metabolic diseases?. Obesity reviews. 2013 Dec;14(12):950-9.

24. Le Chatelier E. Consortium M. 2013. Richness of human gut microbiome correlates with metabolic markers. Nature. 2013;500:541-6.

25. Rittiphairoj T, Pongpirul K, Mueller NT, Li T. Probiotics for glycemic control in patients with type 2 diabetes mellitus: protocol for a systematic review. Systematic reviews. 2019 Dec;8(1):1-6.

26. Senok AC, Ismaeel AY, Botta GA. Probiotics: facts and myths. Clinical Microbiology and Infection. 2005 Dec 1;11(12):958-66.

27. Syngai GG, Gopi R, Bharali R, Dey S, Lakshmanan GA, Ahmed G. Probiotics-the versatile functional food ingredients. Journal of food science and technology. $2016 \mathrm{Feb}$ 1;53(2):921-33.

28. Panahi S, Tremblay A. The potential role of yogurt in weight management and prevention of type 2 diabetes. Journal of the American College of Nutrition. 2016 Nov 16;35(8):717-31.

29. Le Roy T, de Hase EM, Van Hul M, Paquot A, Pelicaen R, Régnier M, Depommier C, Druart C, Everard A, Maiter D, Delzenne NM. Dysosmobacter welbionis is a newly isolated human commensal bacterium preventing diet-induced obesity and metabolic disorders in mice. Gut. 2021 Jun 4.

30. Hampe CS, Roth CL. Probiotic strains and mechanistic insights for the treatment of type 2 diabetes. Endocrine. 2017 Nov;58(2):207-27.

31. Bäckhed, F., Manchester, J., Semenkovich, C., \& Gordon, J. (2007). Mechanisms underlying the resistance to diet-induced obesity in germ-free mice. Proceedings $O f$ The National Academy Of Sciences, 104(3), 979-984. doi: 10.1073/pnas.0605374104

32. Niibo M, Shirouchi B, Umegatani M, Morita Y, Ogawa A, Sakai F, Kadooka Y, Sato M. Probiotic Lactobacillus gasseri SBT2055 improves insulin secretion in a diabetic rat model. Journal of dairy science. 2019 Feb 1;102(2):997-1006.

33. Tanti JF, Ceppo F, Jager J, Berthou F. Implication of inflammatory signaling pathways in obesity-induced insulin resistance. Frontiers in endocrinology. 2013 Jan 8;3:181.

34. Orr JS, Puglisi MJ, Ellacott KL, Lumeng CN, Wasserman DH, Hasty AH. Toll-like receptor 4 deficiency promotes the alternative activation of adipose tissue macrophages. Diabetes. 2012 Nov 1;61(11):2718-27.

35. Mogensen TH. Pathogen recognition and inflammatory signaling in innate immune defenses. Clinical microbiology reviews. 2009 Apr;22(2):240-73.

36. Kang JH, Yun SI, Park MH, Park JH, Jeong SY, Park HO. Anti-obesity effect of Lactobacillus gasseri BNR17 in high-sucrose diet-induced obese mice. PloS one. 2013 Jan 30;8(1):e54617. 
Table 1. Effect of probiotic supplementation on glucose metabolism and IR

\begin{tabular}{|c|c|c|c|c|c|}
\hline Reference & $\begin{array}{l}\text { Diet and Experimental } \\
\text { groups }\end{array}$ & Probiotic Type and Dose & $\begin{array}{l}\text { Duration of } \\
\text { Treatment }\end{array}$ & $\begin{array}{l}\text { Glucose and Insulin } \\
\text { Profile }\end{array}$ & Other Effects \\
\hline Yadav et al. 2013 & HFD (Non-Diabetic) & $\begin{array}{l}\text { VSL\#3 (L. acidophilus MB } 443, L . \\
\text { delbrueckii subsp. bulgaricus } \mathrm{MB} 453, L \text {. } \\
\text { casei MB } 451, \text { L. plantarum } \mathrm{MB} 452, B . \\
\text { longum } \mathrm{Y} 10, B \text {. infantis } \mathrm{Y} 1, B . \text { breve } \mathrm{Y} 8 \text {, } \\
\text { and } S . \text { salivarius subsp. thermophilus } \mathrm{MB} \\
455 \text { ) } \\
5 \mathrm{mg} / \mathrm{kg} \text { of body weight }\end{array}$ & $\begin{array}{l}\text { Preventive } \\
\text { Treatment: } \\
8 \text { weeks. } \\
\text { Therapeutic } \\
\text { Treatment: } \\
13 \text { weeks on } \\
\text { HFD+8 weeks } \\
\text { of Probiotic } \\
\text { treatment }\end{array}$ & $\begin{array}{l}\uparrow \text { Insulin Tolerance } \\
\uparrow \text { Glucose Tolerance }\end{array}$ & $\begin{array}{l}\downarrow \text { AgRP, } \downarrow \text { NPY, } \uparrow \text { POMC } \\
\downarrow \text { Adipocyte Size } \\
\downarrow \text { Body Weight Gain } \\
\downarrow \text { Firmicutes } \uparrow \text { Bacteroidetes } \\
\uparrow \text { Bifidobactericeae } \\
\downarrow \text { Pro Inflammatory } \\
\text { Cytokines, TG, FFA }\end{array}$ \\
\hline Everard et al. 2013 & HFD (Non-Diabetic) & $\begin{array}{l}\text { A. muciniphila } \\
2.10^{8} \mathrm{cfu} / \mathrm{day}\end{array}$ & 4 weeks & $\downarrow$ Insulin resistance & $\begin{array}{l}\downarrow \text { Weight gain } \\
\downarrow \text { Endotoxemia } \\
\uparrow \text { Restoration of mucosal } \\
\text { layer thickness }\end{array}$ \\
\hline $\begin{array}{l}\text { Moya-Pérez et al. } \\
2014\end{array}$ & HFD (Non-Diabetic) & $\begin{array}{l}\text { Bifidobacterium } \\
\text { pseudocatenulatum CECT } 7765 \\
10^{9} \mathrm{cfu} / \text { day }\end{array}$ & 7 weeks & $\begin{array}{l}\square \text { Glucose } \\
\square \text { Insulin }\end{array}$ & $\begin{array}{l}\downarrow \text { Leptin levels } \\
\downarrow \text { Serum chol, TG }\end{array}$ \\
\hline Wang et al. 2015 & HFD (Non-Diabetic) & $\begin{array}{l}\text { Lactobacillus paracasei CNCM I-4270 } \\
\text { (LC), L. rhamnosus I-3690 (LR) or } B \text {. } \\
\text { animalis subsp. lactis I-2494 (BA) } \\
10^{8} \text { cells/day (individually, not in } \\
\text { combination) }\end{array}$ & 12 weeks & $\begin{array}{l}\uparrow \text { Glucose-insulin } \\
\text { homeostasis }\end{array}$ & $\begin{array}{l}\downarrow \text { Weight gain } \\
\downarrow \text { Macrophage infiltration } \\
\downarrow \text { TNF- } \alpha \text { expression } \\
\downarrow \text { LBP (a marker of } \\
\text { endotoxin) }\end{array}$ \\
\hline Wang et al., 2015 & $\begin{array}{l}\text { C (Non -Diabetic control) } \\
\text { M (HFD diabetic control) } \\
\mathrm{P}(\mathrm{HFD}+\text { pioglitazone }) \\
10^{\wedge} 8(\mathrm{HFD}+L . \\
\left.\text { casei } 10^{\wedge} 8\right) \\
10^{\wedge} 9(\mathrm{HFD}+L . \\
\left.\text { casei } 10^{\wedge} 9\right) \\
10^{\wedge} 10(\mathrm{HFD}+L . \\
\text { casei } 10^{\wedge} 1\end{array}$ & $\begin{array}{l}\text { Lactobacillus casei } C \text { CFM419 } \\
10^{\wedge} 8 \text { or } 10^{\wedge} 9 \text { or } 10^{\wedge} 10 \mathrm{CFU}\end{array}$ & 12 weeks & $\begin{array}{l}\text { Treatment induced- } \\
\text {-Glucose AUC } \\
\text {-Insulin level } \\
\text {-HOMA-IR } \\
\mathrm{Pb} \text { doses of } 10^{\wedge} 8 \text { - } \\
10^{\wedge} 9 \mathrm{CFU} \text { showed } \\
\text { better improvement in } \\
\text { insulin resistance than } \\
10^{\wedge} 10\end{array}$ & $\begin{array}{l}\text { Probiotic groups- } \\
- \text { TNF- } \alpha \\
\uparrow \text { protective effects in islet } \\
\text { cells. } \\
\uparrow \text { Acetate, butyrate and GLP- } \\
\text { at } 10^{\wedge} 9 \text { and } 10^{\wedge} 10 \mathrm{CFU} \\
\text { doses } \\
{ }^{-} \text {Firmicutes-to- } \\
\text { Bacteroidetes ratio. }\end{array}$ \\
\hline
\end{tabular}




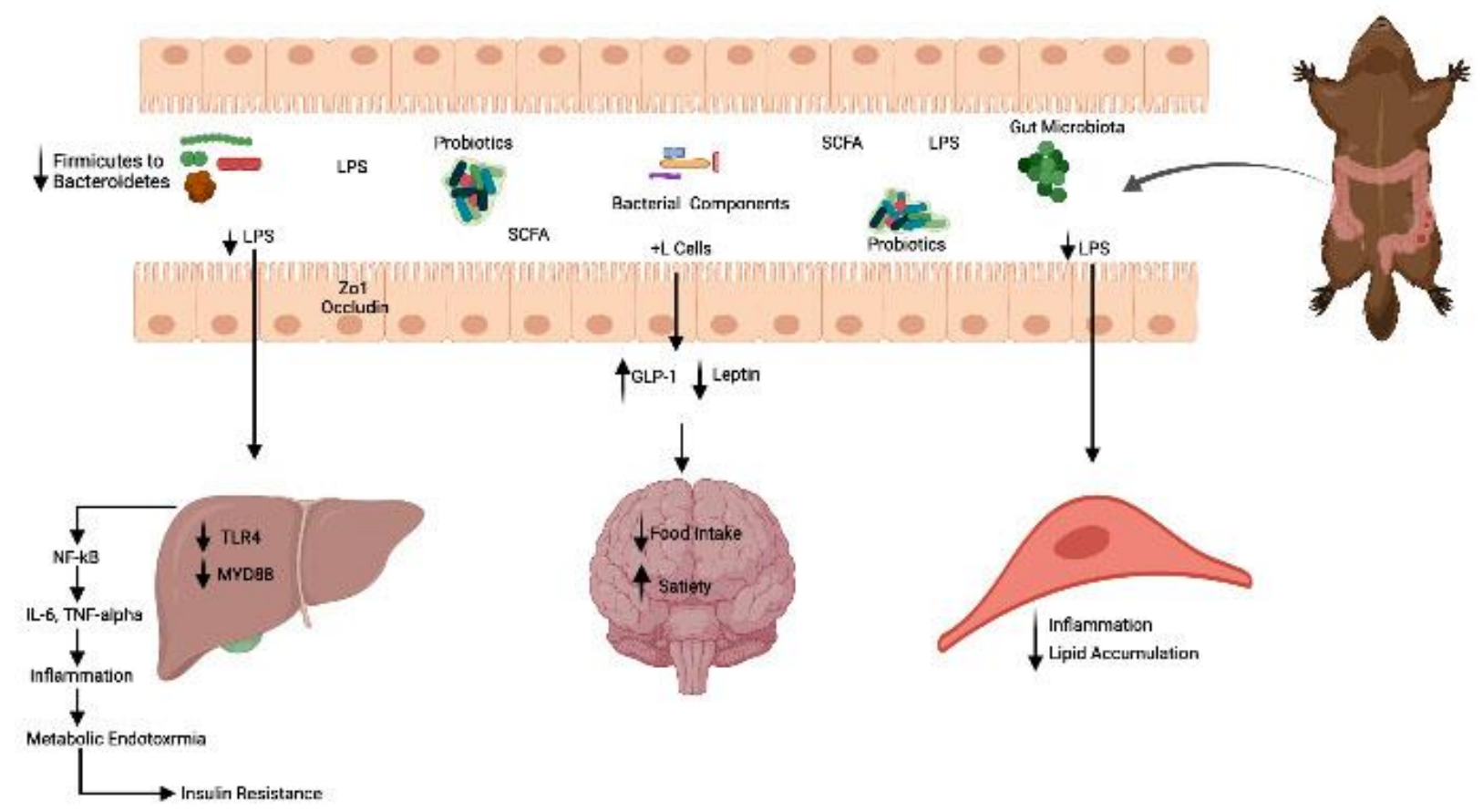

Figure 1. Effects of probiotic supplementation on gut microbiota and intestinal barrier integrity. Increased expression of TJ proteins Zo-1 and Occludin, reduced translocation of LPS ameliorating metabolic endotoxiaemia and insulin resistance. Increased GLP-1 and decreased release of leptin affects hypothalamus causing deceased food intake. Probiotics also prevent gut dysbiosis and decrease Firmicutes to Bacteroidete ratio. In muscle cells, inflammation and lipid accumulation is reduced. 